\title{
Increasing Prevalence of Gastroschisis — 14 States, 1995-2012
}

\begin{abstract}
Abbey M. Jones, $\mathrm{MPH}^{1}$; Jennifer Isenburg, $\mathrm{MSPH}^{1}$; Jason L. Salemi, $\mathrm{PhD}^{2}$; Kathryn E. Arnold, $\mathrm{MD}^{1}$; Cara T. Mai, DrPH${ }^{1}$; Deepa Aggarwal, PhD 3 ; William Arias, $\mathrm{MPH}^{4}$; Gerard E. Carrino, $\mathrm{PhD}^{5}$; Emily Ferrell, $\mathrm{MPH}^{6}$; Olakunle Folorunso, MBBS7 ; Brendan Ibe, MD ${ }^{8}$; Russell S. Kirby, PhD ${ }^{9}$; Heidi R. Krapfl, MS ${ }^{10}$; Lisa K. Marengo, MS ${ }^{11}$; Bridget S. Mosley, MPH ${ }^{12}$; Amy E. Nance, MPH ${ }^{13}$; Paul A. Romitti, PhD ${ }^{14}$; Joseph Spadafino, MPH ${ }^{15}$; Jennifer Stock ${ }^{16}$; Margaret A. Honein, $\mathrm{PhD}^{1}$
\end{abstract}

Gastroschisis is a serious congenital defect in which the intestines protrude through an opening in the abdominal wall. Gastroschisis requires surgical repair soon after birth and is associated with an increased risk for medical complications and mortality during infancy. Reports from multiple surveillance systems worldwide have documented increasing prevalence of gastroschisis since the 1980s, particularly among younger mothers $(1,2)$; however, since publication of a multistate U.S. report that included data through 2005 (1), it is not known whether prevalence has continued to increase. Data on gastroschisis from 14 population-based state surveillance programs were pooled and analyzed to assess the average annual percent change (AAPC) in prevalence and to compare the prevalence during 2006-2012 with that during 1995-2005, stratified by maternal age and race/ethnicity. The pooled data included approximately $29 \%$ of U.S. births for the period 1995-2012. During 1995-2012, gastroschisis prevalence increased in every category of maternal age and race/ethnicity, and the AAPC ranged from $3.1 \%$ in non-Hispanic white (white) mothers aged $<20$ years to $7.9 \%$ in non-Hispanic black (black) mothers aged $<20$ years. These corresponded to overall percentage increases during 1995-2012 that ranged from $68 \%$ in white mothers aged $<20$ years to $263 \%$ in black mothers aged $<20$ years. Gastroschisis prevalence increased 30\% between the two periods, from 3.6 per 10,000 births during $1995-2005$ to 4.9 per 10,000 births during 2006-2012 (prevalence ratio $=1.3,95 \%$ confidence interval [CI]: $1.3-1.4$ ), with the largest increase among black mothers aged $<20$ years (prevalence ratio $=2.0$, 95\% CI: 1.6-2.5). Public health research is urgently needed to identify factors contributing to this increase.

To follow up on a study that included gastroschisis prevalence data from 15 states and reported a near doubling of gastroschisis prevalence during 1995-2005 (1), CDC requested updated data from each of these states for 1995-2012. Fourteen states* provided data on gastroschisis cases crosstabulated by maternal age groups ( $<20$ years, $20-24$ years, $25-29$ years, 30-34 years, and $\geq 35$ years) and race/ethnicity (white, black, and Hispanic,

\footnotetext{
* The 14 states that provided data on gastroschisis and the years for which data were provided were Arizona (1995-2012), Arkansas (1995-2012), California (1995-2012), Colorado (1997-2012), Georgia (1995-2012), Iowa (19952012), Kentucky (1998-2012), New Mexico (1998-2012), New York (1995-2012), North Carolina (1999-2012), Oklahoma (1995-2012), Rhode Island (2002-2012), Texas (1996-2012), and Utah (1997-2012).
}

with all other racial/ethnic groups included in the total). The gastroschisis case definition was based on the British Pediatric Association Classification of Diseases code (756.71) or the International Classification of Diseases, Ninth Revision, Clinical Modification (ICD-9-CM) code for gastroschisis (756.73, or before $10 / 1 / 2009,756.79$, with verification to confirm cases of gastroschisis, because the previous code was shared with omphalocele). Gastroschisis cases included live births, fetal deaths, ${ }^{\dagger}$ and elective terminations. ${ }^{\S}$

Data were pooled at CDC, and gastroschisis prevalence was calculated for each year, maternal age group, and race/ethnicity. Prevalence was calculated as number of gastroschisis cases among all birth outcomes divided by the total number of live births. The denominators of total number of live births in the same catchment area as the birth defects surveillance program were reported by states or obtained from public use data files. Poisson exact methods were used to calculate 95\% CIs for each prevalence estimate. Prevalence ratios were calculated by dividing the prevalence during 2006-2012 by the prevalence during 1995-2005, and CIs for the prevalence ratios were calculated using Poisson regression.

Because the comparison of prevalence between the two study periods involved an artificial breakpoint during the 18-year data span and only examined pooled prevalence within those periods, joinpoint regression analysis was used to identify statistically significant changes in the annual prevalence of gastroschisis over the course of the entire study period (1995-2012). Joinpoint regression initially models annual trend data by fitting a straight line (i.e., zero joinpoints). Then, joinpoints are added, one at a time, and a Monte Carlo permutation test is used to determine the optimal number of joinpoints. Each joinpoint in the final model corresponds to a significant change in the trend, and an AAPC and its $95 \% \mathrm{CI}$ are calculated to describe how the rate changes within each time interval (3). The estimated overall percent change was calculated by first converting the AAPC to the projected single year change in prevalence and then exponentiating to the number of years studied minus one to estimate the total increase throughout the 18 years. This gives the magnitude of the increase, which

\footnotetext{
${ }^{\dagger}$ Fetal deaths were not reported from Rhode Island, Kentucky during 1998-2003, or New York during 2008-2012.

$\$$ Elective terminations were not reported from Arizona, Colorado, Kentucky, New York, North Carolina, and Rhode Island.
} 
is then converted to a percent increase. For example, an AAPC of 5 represents a projected single year change of 1.05, which would correspond to a 2.29-fold increase over the entire study period $\left(1.05^{\wedge} 17=2.29\right)$. This corresponds to an overall change of $129 \%$ ([2.29-1] x 100). The Joinpoint Regression Program, version 4.2.0 (National Cancer Institute), was used to conduct joinpoint regression.

During 1995-2005, 4,369 gastroschisis cases were detected among 12,014,244 live births (prevalence $=3.6$ per 10,000 live births, 95\% CI: 3.5-3.7), and during 2006-2012, 4,497 gastroschisis cases were detected among 9,264,540 live births (prevalence $=4.9$ per 10,000 live births, 95\% CI: 4.7-5.0). Comparing the two periods, gastroschisis prevalence increased $30 \%$ (prevalence ratio $=1.3,95 \% \mathrm{CI}: 1.3-1.4)$ during 2006-2012 compared with 1995-2005. The prevalence of gastroschisis increased over the course of the study period in each of the five maternal age groups (Figure).
Because fewer cases were detected among older maternal age groups, maternal age was collapsed into three groups $(<20$ years, $20-24$ years, and $\geq 25$ years) to stratify the data simultaneously by maternal age and race/ethnicity. A significant increase in gastroschisis prevalence occurred in each maternal age group from the first period (1995-2005) to the second (2006-2012) (Table). Statistically significant increases comparing the two periods were seen in eight of the nine categories of maternal age and race/ethnicity assessed. Among mothers aged $<20$ years and 20-24 years, significant increases were seen in all racial/ ethnic groups examined. The prevalence of gastroschisis during 2006-2012 among mothers aged $<20$ years was higher among white mothers (18.1 per 10,000 live births, 95\% CI: 16.7-19.7) and Hispanic mothers (16.1 per 10,000 live births, 95\% CI: 14.9-17.3) than among black mothers (10.2 per 10,000 live births, 95\% CI: 8.7-11.9); however, black mothers in this age group experienced the largest increase in prevalence,

FIGURE. Trends in gastroschisis prevalence, by maternal age group - 14 states,* 1995-2012

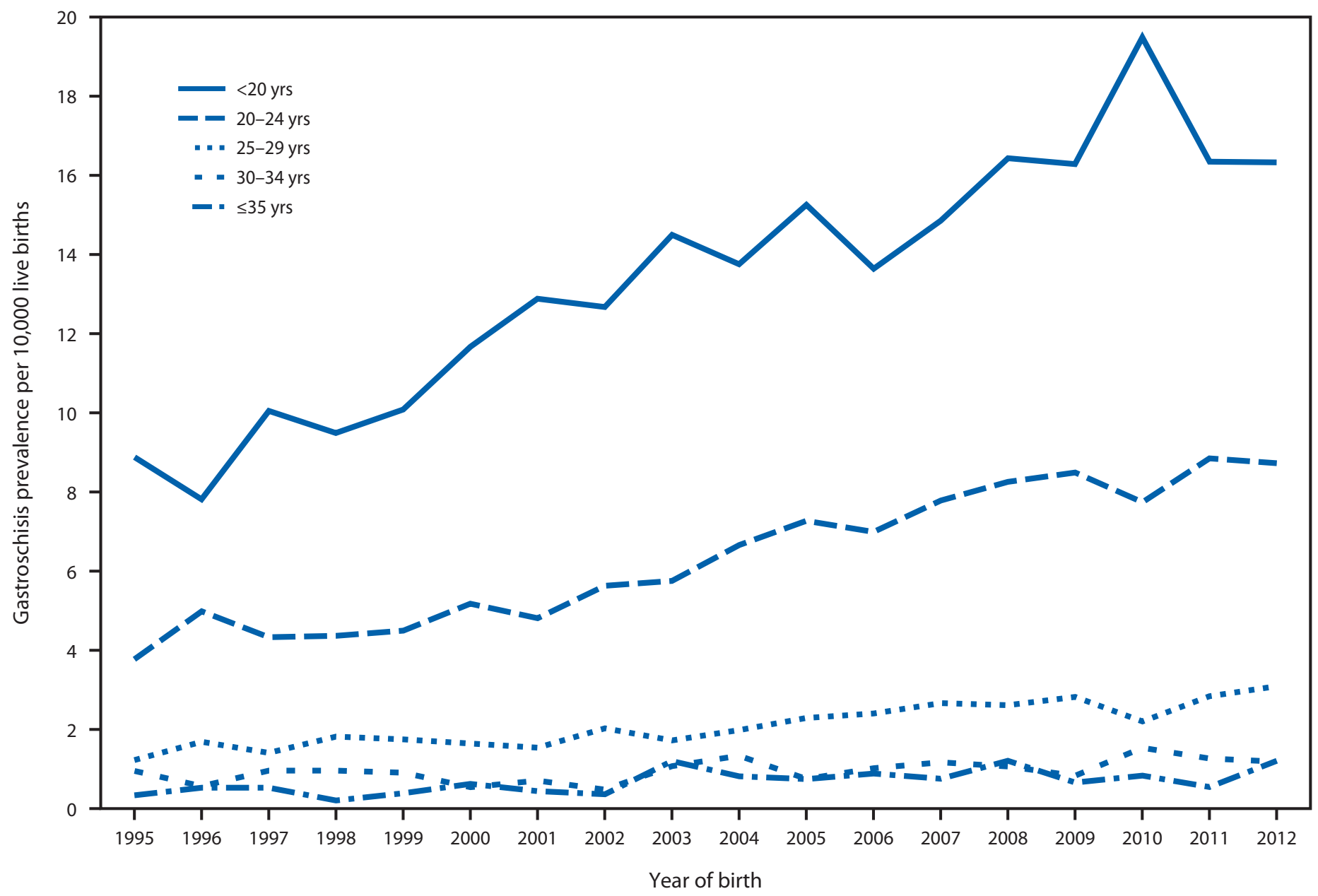

* States contributing data and years for which data are provided: Arizona (1995-2012), Arkansas (1995-2012), California (1995-2012), Colorado (1997-2012), Georgia (1995-2012), lowa (1995-2012), Kentucky (1998-2012), New Mexico (1998-2012), New York (1995-2012), North Carolina (1999-2012), Oklahoma (1995-2012), Rhode Island (2002-2012), Texas (1996-2012), Utah (1997-2012). Total live births = 21,278,784. 
which doubled between the two study periods (prevalence ratio $=2.0,95 \%$ CI: 1.6-2.5).

Using Joinpoint regression to assess temporal trends within strata of maternal age and race/ethnicity, the AAPC indicated a significant increase in prevalence for all nine groups (Table). Zero joinpoints were identified for each of the nine strata, indicating that a single AAPC estimate was appropriate for the entire study period for each group. The two highest AAPCs among these strata were in black mothers aged $<20$ years and 20-24 years. The AAPCs ranged from a low of 3.1 in white mothers aged $<20$ years to a high of 7.9 in black mothers aged $<20$ years. These corresponded to overall percent increases from 1995 to 2012 that ranged from $68 \%$ in white mothers aged $<20$ years to $263 \%$ in black mothers aged $<20$ years.

\section{Discussion}

The prevalence of gastroschisis increased significantly during the study period, and prevalence has continued to increase beyond 2005, the end of the period included in the previous multistate report (1). Gastroschisis is associated with young maternal age, with the highest prevalence among mothers aged $<20$ years; however, significant increases in prevalence were seen in all age groups during 2006-2012 compared with 1995-2005. The greatest increases in prevalence occurred among younger, black mothers, but the prevalence in black mothers remains lower than in white and Hispanic mothers. Joinpoint analyses demonstrated a steady and significant increase in prevalence across all assessed categories of maternal age and race/ethnicity.
Increases in gastroschisis prevalence have been reported both in the United States and internationally, but the current evidence has not led to the identification of the underlying cause or causes of these increases $(1,2)$. The association between young maternal age and gastroschisis was first reported in the late 1970s, and this risk factor has been documented consistently in subsequent studies (4-6). However, the increased prevalence of gastroschisis is not because of an increase in teen births, which have declined in recent years, or to a change in the distribution of births to teen mothers, as birth rates have decreased among women of all ages $<20$ years (7). Investigators in Norway reported an independent association of young paternal age with gastroschisis, after accounting for maternal age (๑). In addition, year of delivery, mother's year of birth, and father's year of birth were each significantly associated with increasing gastroschisis prevalence from 1967 to 1998 (6). Epidemiologic patterns indicate that lifestyle behaviors, environmental exposures, or other risk factors disproportionately affecting young women might play a role. A 2008 review noted that risk factors associated with gastroschisis, after adjusting for maternal age, have included lower socioeconomic status, lower body mass index and other indicators of poor nutrition (lower intake of high quality nutrients and dietary fats), smoking, use of illicit drugs, alcohol, or analgesic medications, and genitourinary infections (5). Additionally, among multiparous and multigravida mothers, a change in paternity since the previous pregnancy has been associated with gastroschisis (5). Studies have also indicated possible age-specific associations between gastroschisis and prior pregnancy loss (8), as well as with certain infections (9).

TABLE. Number of cases and gastroschisis prevalence, prevalence ratio, and average annual percent change, by maternal age group and race/ethnicity 14 states, ${ }^{*} 1995-2012$

\begin{tabular}{|c|c|c|c|c|c|c|c|c|}
\hline \multirow{2}{*}{$\begin{array}{l}\text { Maternal } \\
\text { age group } \\
\text { (yrs) }{ }^{\dagger}\end{array}$} & \multirow[b]{2}{*}{$\begin{array}{c}\text { Maternal } \\
\text { race/ethnicity }\end{array}$} & \multicolumn{2}{|r|}{ 1995-2005 } & \multicolumn{2}{|r|}{ 2006-2012 } & \multirow[b]{2}{*}{$\mathrm{PR}^{\text {ศी }}(95 \% \mathrm{Cl})$} & \multicolumn{2}{|c|}{ Joinpoint analysis } \\
\hline & & No. & $\begin{array}{l}\text { Prevalence } \\
(95 \% \mathrm{CI})\end{array}$ & No. & $\begin{array}{l}\text { Prevalence } \\
(95 \% \mathrm{Cl})\end{array}$ & & $\begin{array}{l}\text { Average annual percent } \\
\text { change }\left(95 \% \mathrm{Cl}^{* *}\right)\end{array}$ & $\begin{array}{l}\text { Overall percent } \\
\text { change }^{t+}\end{array}$ \\
\hline \multirow[t]{4}{*}{$<20$} & Non-Hispanic white & 816 & $15.4(14.3-16.5)$ & 574 & $18.1(16.7-19.7)$ & $1.2(1.1-1.3)$ & $3.1(1.5-4.7)$ & 68 \\
\hline & Non-Hispanic black & 137 & $5.2(4.3-6.1)$ & 169 & $10.2(8.7-11.9)$ & $2.0(1.6-2.5)$ & $7.9(5.7-10.1)$ & 263 \\
\hline & Hispanic & 698 & $11.6(10.7-12.5)$ & 710 & $16.1(14.9-17.3)$ & $1.4(1.2-1.5)$ & $4.4(3.1-5.7)$ & 108 \\
\hline & Total§§ & 1,749 & $11.9(11.3-12.4)$ & 1,562 & $16.1(15.3-16.9)$ & $1.4(1.3-1.5)$ & $4.3(3.3-5.3)$ & 105 \\
\hline \multirow[t]{4}{*}{$20-24$} & Non-Hispanic white & 846 & $6.0(5.6-6.4)$ & 889 & $8.8(8.2-9.4)$ & $1.5(1.3-1.6)$ & $4.6(3.5-5.7)$ & 115 \\
\hline & Non-Hispanic black & 135 & $2.9(2.4-3.4)$ & 193 & $5.4(4.7-6.2)$ & $1.9(1.5-2.3)$ & $7.0(3.7-10.4)$ & 216 \\
\hline & Hispanic & 601 & $5.5(5.1-6.0)$ & 706 & $8.3(7.7-8.9)$ & $1.5(1.3-1.7)$ & $5.6(4.3-6.8)$ & 151 \\
\hline & Total $\S \S$ & 1,687 & $5.3(5.1-5.6)$ & 1,899 & $8.1(7.7-8.4)$ & $1.5(1.4-1.6)$ & $5.2(4.4-6.0)$ & 137 \\
\hline \multirow[t]{4}{*}{$\geq 25$} & Non-Hispanic white & 501 & $1.2(1.1-1.3)$ & 541 & $1.7(1.6-1.9)$ & $1.4(1.3-1.6)$ & $4.1(2.6-5.6)$ & 99 \\
\hline & Non-Hispanic black & 71 & $0.9(0.7-1.1)$ & 77 & $1.2(1.0-1.5)$ & $1.4(1.0-1.9)$ & $3.5(0.4-6.8)$ & 81 \\
\hline & Hispanic & 213 & $1.2(1.0-1.3)$ & 314 & $1.9(1.7-2.1)$ & $1.6(1.3-1.9)$ & $4.7(2.4-7.2)$ & 120 \\
\hline & Total§§ & 853 & $1.2(1.1-1.2)$ & 1,014 & $1.7(1.6-1.8)$ & $1.5(1.3-1.6)$ & $4.5(3.5-5.4)$ & 111 \\
\hline
\end{tabular}

Abbreviations: $\mathrm{Cl}$ = confidence interval, $\mathrm{PR}=$ prevalence ratio.

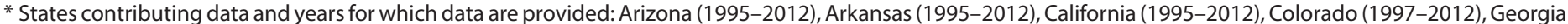

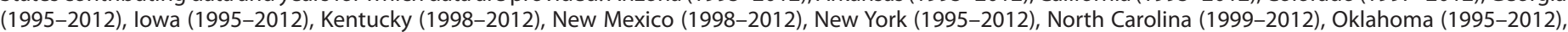
Rhode Island (2002-2012), Texas (1996-2012), Utah (1997-2012). Total live births = 21,278,784.

† Cases missing information on maternal age are not included in this table.

§ Prevalence per 10,000 live births.

I Prevalence during 2006-2012 divided by the prevalence during 1995-2005.

** Statistically significant differences from zero percent change.

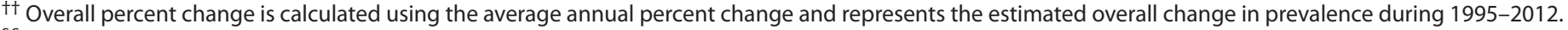

$\S \S$ Total includes non-Hispanic white, non-Hispanic black, Hispanic, all other reported racial/ethnic groups and other/unknown maternal race/ethnicity. 


\section{Summary}

What is already known on this topic?

Gastroschisis is strongly associated with young maternal age, and a previous U.S. report indicated that the prevalence of gastroschisis nearly doubled from 1995 to 2005.

What is added by this report?

Gastroschisis prevalence has increased for all maternal age groups. Significant increases as measured by the average annual percent change were observed for all assessed categories of maternal age and race/ethnicity. The largest estimated increase over the 18 year period ( $263 \%$ overall percent change) was observed for non-Hispanic black mothers aged $<20$ years.

What are the implications for public health practice?

The observed increases in gastroschisis prevalence are not explained by demographic changes in maternal age or race/ ethnicity. Public health research is urgently needed to identify the causal factor(s) contributing to this increase.

The findings in this report are subject to at least three limitations. First, because no information on risk factors other than maternal age and race/ethnicity was requested from state surveillance programs, potential causes for the increase in prevalence could not be examined. Second, not all states were able to provide data as far back as 1995 . However, incomplete data for the earlier years of the study is unlikely to affect the results. Because data were pooled for 1995-2005, the prevalence estimates for that earlier study period are more heavily influenced by the later years of that time frame. Previous research has demonstrated an increase in prevalence from 1995 to 2005; therefore, the missing data from earlier years in this study is likely to result in prevalence ratios that are biased slightly and conservatively toward the null. Finally, it is possible that the increase in prevalence could be due to improved ascertainment of gastroschisis cases over time. However, this is unlikely because gastroschisis is immediately apparent at birth. Additionally, omphalocele, a defect that has a similar presentation at birth and previously shared an ICD-9-CM code with gastroschisis, is not increasing in prevalence, making it implausible that the increase observed is due to any confusion between these defects (10).

These findings have implications for prioritizing public health research on gastroschisis to identify factors contributing to the high risk associated with young maternal age and factors associated with the increasing prevalence over the past 20 years. Gastroschisis is unusual among birth defects in that it disproportionately affects younger mothers, a vulnerable population. The continued increase in age-adjusted prevalence and the pace of the increase suggests that unidentified risk factors might be contributing. Identification of these risk factors is needed to inform public health interventions and reduce prevalence. Ongoing surveillance is essential to monitor any further increases in prevalence.

\section{Acknowledgments}

Arizona Birth Defects Monitoring Program; Arkansas Reproductive Health Monitoring System; California Birth Defects Monitoring Program; Colorado Responds to Children with Special Needs Section; Iowa Registry for Congenital and Inherited Disorders; Kentucky Birth Surveillance Registry; Metropolitan Atlanta Congenital Defects Program, Atlanta, Georgia; New Mexico Birth Defects Prevention and Surveillance System; New York State Congenital Malformations Registry; North Carolina Birth Defects Monitoring Program; Oklahoma Birth Defect Registry; Rhode Island Birth Defects Surveillance Program; Texas Birth Defects Epidemiology and Surveillance Branch; Utah Birth Defect Network.

\footnotetext{
${ }^{1}$ Birth Defects Branch, National Center on Birth Defects and Developmental Disabilities, CDC; ${ }^{2}$ Baylor College of Medicine, Houston, Texas; ${ }^{3}$ California Birth Defects Monitoring Program, Maternal, Child and Adolescent Health Program, California Department of Public Health; ${ }^{4}$ Rhode Island Department of Health; ${ }^{5}$ March of Dimes Foundation, White Plains, New York; ${ }^{6}$ Kentucky Department for Public Health; ${ }^{7}$ Oklahoma State Department of Health; ${ }^{8}$ Georgia Department of Public Health; ${ }^{9}$ College of Public Health, University of South Florida; ${ }^{10}$ New Mexico Department of Health; ${ }^{11}$ Birth Defects Epidemiology and Surveillance Branch, Texas Department of State Health Services; ${ }^{12}$ Arkansas Reproductive Health Monitoring System, Arkansas Children's Hospital Research Institute; ${ }^{13}$ Utah Birth Defect Network, Division of Family Health and Preparedness, Children with Special Health Care Needs Bureau, Utah Department of Health; ${ }^{14}$ Iowa Registry for Congenital and Inherited Disorders; ${ }^{15}$ Arizona Department of Health Services; ${ }^{16}$ North Carolina Division of Public Health.

Corresponding author: Abbey M. Jones, amjones1@cdc.gov, 404-498-3018.
}

\section{References}

1. Kirby RS, Marshall J, Tanner JP, et al.; National Birth Defects Prevention Network. Prevalence and correlates of gastroschisis in 15 states, 1995 to 2005. Obstet Gynecol 2013;122:275-81. http://dx.doi.org/10.1097/ AOG.0b013e31829cbbb4.

2. Castilla EE, Mastroiacovo P, Orioli IM. Gastroschisis: international epidemiology and public health perspectives. Am J Med Genet C Semin Med Genet 2008;148C:162-79. http://dx.doi.org/10.1002/ajmg.c.30181.

3. Kim HJ, Fay MP, Feuer EJ, Midthune DN. Permutation tests for joinpoint regression with applications to cancer rates. Stat Med 2000;19:335-51.

4. Colombani PM, Cunningham MD. Perinatal aspects of omphalocele and gastroschisis. Am J Dis Child 1977;131:1386-8.

5. Rasmussen SA, Frías JL. Non-genetic risk factors for gastroschisis. Am J Med Genet C Semin Med Genet 2008;148C:199-212. http://dx.doi. org/10.1002/ajmg.c.30175.

6. Kazaura MR, Lie RT, Irgens LM, et al. Increasing risk of gastroschisis in Norway: an age-period-cohort analysis. Am J Epidemiol 2004;159:358-63. http://dx.doi.org/10.1093/aje/kwh051.

7. Ventura SJ, Hamilton BE, Mathews TJ. National and state patterns of teen births in the United States, 1940-2013. Natl Vital Stat Rep 2014;63:1-34.

8. Rittler M, Campaña H, Ermini ML, et al. Gastroschisis and young mothers: what makes them different from other mothers of the same age? Birth Defects Res A Clin Mol Teratol 2015;103:536-43. http:// dx.doi.org/10.1002/bdra.23374.

9. Feldkamp ML, Reefhuis J, Kucik J, et al. Case-control study of self reported genitourinary infections and risk of gastroschisis: findings from the national birth defects prevention study, 1997-2003. BMJ 2008;336:1420-3. http://dx.doi.org/10.1136/bmj.39567.509074.25.

10. Marshall J, Salemi JL, Tanner JP, et al.; National Birth Defects Prevention Network. Prevalence, correlates, and outcomes of omphalocele in the United States, 1995-2005. Obstet Gynecol 2015;126:284-93. http:// dx.doi.org/10.1097/AOG.0000000000000920. 